\title{
MMP13 is a critical target gene during the progression of osteoarthritis
}

Meina Wang ${ }^{1,2}$, Erik R Sampson ${ }^{1,3}$, Hongting Jin ${ }^{1,4}$, Jia Li ${ }^{5,6}$, Qiao H Ke ${ }^{5}$, Hee-Jeong $\mathrm{Im}^{5}$ and Di Chen ${ }^{1,5^{*}}$

\begin{abstract}
Introduction: Osteoarthritis (OA) is a degenerative joint disease affecting a large population of people. The mechanism of this highly prevalent disease is not fully understood. Currently there is no effective disease-modifying treatment for OA. The purpose of this study was two-fold: 1) to investigate the role of MMP13 in the development of $\mathrm{OA}$; and 2) to evaluate the efficacy of the MMP13 inhibitor CL82198 as a pharmacologic treatment for preventing OA progression.
\end{abstract}

Methods: To investigate the role of the endogenous Mmp13 gene in OA development, tamoxifen was administered to two-week-old Col2CreER;Mmp13 $3^{\text {fx/fx }}\left(\mathrm{Mmp} 13^{\mathrm{CO} / 2 E R}\right)$ and Cre-negative control mice for five days. OA was induced by meniscal-ligamentous injury (MLI) when the mice were 10 weeks old and MLI or sham-operated joints were harvested 4, 8, 12, or 16 weeks after surgery. To evaluate the efficacy of CL82198, MLI surgery was performed on 10-week-old wild type mice. CL82198 or saline was administered to the mice daily beginning immediately after the surgery for up to 16 weeks. The joint tissues collected from both experiments were evaluated by cartilage grading, histology/ histomorphometry, immunohistochemistry $(\mathrm{HC})$, and terminal deoxynucleotidyl transferase dUTP nick end labeling (TUNEL) staining. The ability of CL82198 to inhibit MMP13 activity in vitro was confirmed by ELISA.

Results: The OA progression was decelerated in Mmp $13^{\text {COILR }}$ mice 8, 12, and 16 weeks post-surgery. Cartilage grading by blinded observers confirmed decreased articular cartilage degeneration in Mmp13 COILER mice at 8,12 and 16 weeks compared to Cre-negative mice. Histomorphometric analysis demonstrated that Mmp13 $3^{\mathrm{CO} / 2 E R}$ mice had a higher articular cartilage area and thickness at 12 and 16 weeks post-surgery compared to the control mice. Results of IHC revealed greater type II collagen and proteoglycan expression in Mmp13 ${ }^{\text {COIZER }}$ mice. Chondrocyte apoptosis, as determined by TUNEL staining, was higher in control mice compared to Mmp13 ${ }^{\text {COI2ER }}$ mice. CL82198 inhibited MMP13 activity in conditioned media from vehicle (> 85\%) or bone morphogenetic protein 2 (BMP2)-treated (> 90\%) primary murine sternal chondrocytes. Intraperitoneal injection of CL82198 decelerated MLI-induced OA progression, increased type II collagen and proteoglycan levels, and inhibited chondrocyte apoptosis compared to saline treatment as determined by OA grading, histology, histomorphometry, IHC, and TUNEL staining, respectively.

Conclusions: Mmp13 is critical for OA progression and pharmacologic inhibition of MMP13 is an effective strategy to decelerate articular cartilage loss in a murine model of injury-induced knee OA.

\section{Introduction}

Osteoarthritis $(\mathrm{OA})$ is a degenerative joint disease and is a major cause of disability. Currently, there is no treatment capable of altering its progression. The major pathological characteristics of OA include progressive loss of articular cartilage, osteophyte formation, and changes in periarticular and subchondral bone [1,2]. The articular

\footnotetext{
* Correspondence: di_chen@rush.edu

'Department of Orthopedics and Rehabilitation, Center for Musculoskeletal Research, University of Rochester Medical Center, Rochester, NY 14642, USA Full list of author information is available at the end of the article
}

cartilage receives most of the attention in OA studies because the primary pathologic feature seen in OA is gross articular cartilage damage.

Matrix metalloproteinase (MMP) 13 is a major enzyme that targets cartilage for degradation. Compared to other MMPs, the expression of MMP13 is more restricted to connective tissue [3]. It not only targets type II collagen in cartilage for degradation, but also degrades proteoglycan, types IV and type IX collagen, osteonectin and perlecan in cartilage [4]. Clinical investigation revealed that patients with articular cartilage destruction have high MMP13

\section{Biomed Central}


expression [5], suggesting that increased MMP13 may be associated with cartilage degradation. Studies have also shown that Mmp13-overexpressing transgenic mice develop a spontaneous OA-like articular cartilage destruction phenotype [6]. The ADAMTS (a disintegrin and metalloproteinase with thrombospondin motifs) family of aggrecanases also contributes to proteoglycan/aggrecan depletion and are associated with cartilage degradation during OA. ADAMTS4 and 5 were identified as the major aggrecanases during OA development. Deletion of the Adamts 5 gene or double knockout of Adamts 4 and Adamts 5 prevented cartilage degradation in a surgically induced murine knee OA model $[7,8]$. These findings indicate that catabolic enzymes play a significant role in $\mathrm{OA}$ progression and support the development of therapies targeting these enzymes as a strategy to decelerate articular cartilage degradation.

Meniscal injuries are among the most common causes of post-traumatic OA in humans. The meniscus is a $\mathrm{C}$-shaped cartilage that functions as a shock-absorbing, load bearing, stability enhancing, and lubricating cushion in the knee joint. Studies show that loss of meniscus integrity and function leads to OA in humans $[9,10]$. The meniscal-ligamentous injury (MLI)-induced murine OA model was initially developed by Clements et al. [11] and this injury model has been further modified and developed in recent studies [12]. This mouse model also permits study of the development of traumainduced $\mathrm{OA}$ in defined genetic backgrounds. In this model, the ligation of the medial collateral ligament coupled with disruption of the meniscus from its anterior-medial attachment induces reproducible OA development over a three-month period [12]. In the present studies, we will determine the role of MMP13 in MLIinduced OA progression. We will use Col2CreER; $M m p 13^{f x / f x}\left(M m p 13^{C o l 2 E R}\right)$ mice to block Mmp13 expression in chondrocytes and use MMP13 inhibitors to inhibit MMP13 activity.

\section{Methods}

Meniscal/ligamentous injury-induced osteoarthritis model and treatment of MMP13 inhibitor

Wild type C57BL/6J and $M m p 13^{\mathrm{fx} / \mathrm{fx}}$ mice [13] were obtained from Jackson Laboratories (Bar Harbor, ME, USA). Col2CreER transgenic mice [14] were crossed with $M m p 13^{\mathrm{fx} / \mathrm{fx}}$ mice to generate Col2CreER;Mmp13 $3^{\mathrm{fx} / \mathrm{fx}}$ mice (referred to hereafter as Mmp13 Col2ER mice) (Table 1). Tamoxifen (Sigma, St. Louis, MO, USA) was administered to two-week-old Mmp13 ${ }^{\text {Col2ER }}$ mice and littermate controls by intraperitoneal (i.p.) injection $(1 \mathrm{mg} / 10 \mathrm{~g}$ body weight) for five days. MLI surgery was performed to induce knee OA [12] in 10-week-old Mmp $13^{\text {Col2ER }}$ and Cre-negative control mice. Details regarding MLI surgery were previously described [12]. The surgery was
Table 1 Breeding of Col2CreER;Mmp13 fx/fx mice (Mmp13 ${ }^{\text {Col2ER }}$ ).

\begin{tabular}{|c|c|}
\hline Breeding & Desired progeny \\
\hline a) Col2CreER $\times M m p 13^{f x / f x}$ & a) Col2CreER;Mmp13 $3^{f x / w t}$ \\
\hline $\begin{array}{l}\text { b) Col2CreER;Mmp13 } 3^{f x / w t} \\
x M m p 13^{f x / f x}\end{array}$ & b) Col2CreER;Mmp13 $3^{f x / f x}$ \\
\hline $\begin{array}{l}\text { c) Col2CreER;Mmp13 } 3^{f x / f x} \\
\text { x Mmp13 } 3^{f x / f x}\end{array}$ & $\begin{array}{l}\text { c) Col2CreER;Mmp } 13^{f x / f x} \text { and } \\
M m p 13^{f x / f x}\end{array}$ \\
\hline
\end{tabular}

performed on the right hind limbs as follows: 1) following anesthesia, making a $5 \mathrm{~mm}$ parapatellar; 2) identifying and transecting the medial collateral ligament with a 25 gauge needle; 3) applying valgus stress to the knee to confirm disruption and provide access to the meniscus; 4 ) detaching and partially removing the anterior horn of the medial meniscus; 5) closing of the wound with 4.0 nylon sutures applied in an interrupted pattern. The left hind limb was used as a control. The left hind limb was opened and the structures of the knee were exposed and then the skin incision closed without manipulating the joint tissue. Preand post-surgery, mice were provided analgesia $(2.5 \mathrm{mg} / \mathrm{kg}$ banamine, i.p. injection) every 24 hours for 72 hours and the sutures were removed after 10 days. Both left and right knee joints were harvested, processed, sectioned and stained $4,8,12$, and 16 weeks post-surgery ( $n=5$ in each group). MLI and sham surgeries were also performed on 10-week-old C57BL/6J mice ( $n=5$ in each group). The MMP13 inhibitor CL82198 (Tocris Bioscience, Minneapolis, MN, USA) was administered to wild type mice beginning one day after surgery by i.p. injection every other day for up to 16 weeks at doses of $1,5,10 \mathrm{mg} / \mathrm{kg}$ body weight. Normal saline was used as a control. Knee joints were collected, sectioned and stained 12 weeks post-surgery $(n=5$ in each group). All protocols were approved by the Institutional Animal Care and Use Committee (IACUC) at the University of Rochester.

\section{Histology and histomorphometry}

Knee joints from each group were harvested and prepared for sectioning and analysis. Samples were fixed in 10\% neutral buffered formalin (VWR, Radnor, PA, USA) for three days, then decalcified with formic acid (Decal Chemical Corp., Suffern, NY, USA) for seven days. After neutralizing with Cal-arrest (Decal Chemical Corp.), samples were processed and embedded in paraffin. Three $\mu \mathrm{m}$ thick mid-saggital sections at three different levels (50 $\mu \mathrm{m}$ apart) were cut from the medial compartment of the joints. The sections were stained with Alcian blue/H\&E $(\mathrm{AB} / \mathrm{H} \& \mathrm{E})$ and safranin $\mathrm{O} /$ Fast Green (SO/FG). Histomorphometric measurements were performed using OsteoMeasure software (OsteoMetrics, Inc., Atlanta, GA, USA). AB/H\&E- or $\mathrm{SO} /$ FG-stained areas were outlined on projected images of each histologic section to determine articular cartilage area and thickness. 


\section{Immunohistochemistry and TUNEL staining}

Three $\mu \mathrm{m}$ thick paraffin sections were baked at $60^{\circ} \mathrm{C}$ overnight. Slides were then deparaffinized, rehydrated, and digested with pepsin. DAKO endogenous blocking reagent (Dako S2003, Carpinteria, CA, USA) was then used to quench endogenous peroxidase for 10 minutes. Normal horse serum or normal goat serum (Vector S-2000, Burlingame, CA, USA) was used to block non-specific binding sites for 20 minutes. Collagen II or collagen X primary antibodies (Thermo Scientific, Rockford, IL, USA) were added and the slides were incubated at $4{ }^{\circ} \mathrm{C}$ overnight. Secondary biotinylated horse anti-mouse antibody (Vector BA-2000) or goat anti-mouse antibody (Vector BA-9200) was added for 30 minutes on the second day, followed by incubation with streptavidin (Pierce 21130, Rockford, IL, USA) for 30 minutes. Positive staining was detected by Romulin AEC Chromagen (Biocare Medical RAEC810L, Concord, CA, USA). To detect chondrocyte apoptosis after meniscal surgery, terminal deoxynucleotidyl transferase dUTP nick end labeling (TUNEL) staining was performed using a kit based on the manufacturer's instruction (Promega, Fitchburg, WI, USA).

\section{Grading of cartilage structure}

Tissue sections were stained with Alcian blue/Orange G and graded by two blinded observers based on the scoring system developed by Chambers et al. [7]. In brief, each section was assigned a grade as follows: $0=$ normal cartilage, that is, lack of superficial zone fibrillation or clefting; $1=$ mild superficial fibrillation; 2 = fibrillation and $/$ or clefting extending below the superficial zone; $3=$ mild $(<20 \%)$ loss of articular cartilage; $4=$ moderate $(20 \%$ to $80 \%$ ) loss of articular cartilage; 5 = focal loss of cartilage to the subchondral bone (eburnation); $6=$ severe $(>80 \%)$ loss of articular cartilage. The progression of OA in different groups was evaluated using this method. Three slides of each sample were analyzed and five mice were used in each group.

\section{MMP13 activity assay}

The ability of CL82198 to inhibit MMP13 activity in vitro was determined using the SensoLyte 520 MMP13 Assay Kit (AnaSpec, Fremont, CA, USA). Five ng of MMP13 and $10 \mu \mathrm{g} / \mathrm{mL}$ of CL82198, or control substrate were added into the MMP13 Assay Kit. MMP13 activity was detected following the manufacturer's instructions. Primary sternal chondrocytes were isolated from three-day-old wild-type (WT) pups as previously described [15]. Cells were plated in 12-well-plates and treated with bone morphogenetic protein 2 (BMP-2) $(100 \mathrm{ng} / \mathrm{ml})$ alone or BMP-2 with the MMP-13 inhibitor CL82198 (1, 5 and $10 \mu \mathrm{M})$ for 60 hours. The culture media were collected and MMP13 activity was determined using the SensoLyte 520 MMP13
Assay Kit (AnaSpec) following the manufacturer's instructions.

\section{Statistical analysis}

Results of all quantitative assays involving multiple doses and time points were analyzed using analysis of variance (ANOVA) followed by Dunnett's test. For experiments comparing two groups, unpaired student's $t$-test was applied. $P<0.05$ was considered to be a significant difference.

\section{Results}

\section{OA progression was decelerated in $M m p 13^{\text {Col2ER }}$ mice}

To investigate if Mmp13 deletion could prevent or decelerate MLI-induced OA, we crossed Col2CreER transgenic mice [14,16] with $M m p 13^{\mathrm{fx} / \mathrm{fx}}$ mice [13] to generate Col2CreER;Mmp13 ${ }^{\mathrm{fx} / \mathrm{fx}}$ mice $\left(M m p 13^{\text {Col2ER }}\right)$ (Table 1). Deletion of the Mmp13 gene in chondrocytes had no significant effect on articular and growth plate chondrocyte morphology [see Additional file 1]. Tamoxifen was administered (i.p., five days) to two-week-old $M m p 13^{\text {Col2ER }}$ mice and had no effect on articular and growth plate cartilage [see Additional file 1]. MLI surgery was performed when the mice were 10-weeks-old to induce OA. Knee joints were harvested $4,8,12$, and 16 weeks post-surgery ( $n=5$ in each group). Histology showed that four weeks post-MLI surgery, articular cartilage was nearly normal in both $M m p 13^{\text {Col2ER }}$ and Cre-negative control groups (Figure 1A). OA-like fibrillation, clefting and cartilage loss down to the tidemark appeared 8 weeks post-surgery and worsened at the 12- and 16-week time points in Crenegative control mice. In $M m p 13^{\text {Col2ER }}$ mice, however, there was markedly less articular cartilage excavation, especially at the 12- and 16-week time points (Figure 1A). OA grading likewise revealed significantly reduced cartilage degeneration at 8,12 and 16 weeks post-surgery in $M m p 13^{\text {Col2ER }}$ mice compared to the control group (Figure 1B). To quantify OA progression, we performed histomorphometry using the OsteoMeasure system. The results showed that articular cartilage area and articular cartilage thickness at proximal tibiae, total cartilage area, and total cartilage thickness progressively decreased at 8,12 , and 16 weeks post-surgery (Figure $1 \mathrm{C}$-F), but this progression was decelerated in $M m p 13^{\operatorname{Col} 2 E R}$ mice compared to the control group. Articular cartilage area and articular cartilage thickness at proximal tibiae were significantly greater at 12 and 16 weeks post-surgery in $\mathrm{Mmp} 13^{\text {Col2ER }}$ groups (Figure 1C-D). Total cartilage area was significantly different at 16 weeks post-surgery (Figure 1E), and total cartilage thickness was significantly different at 8,12 , and 16 weeks post-surgery (Figure 1F). Articular cartilage area and thickness at distal femora had no significant changes at any time point post-surgery. 
A
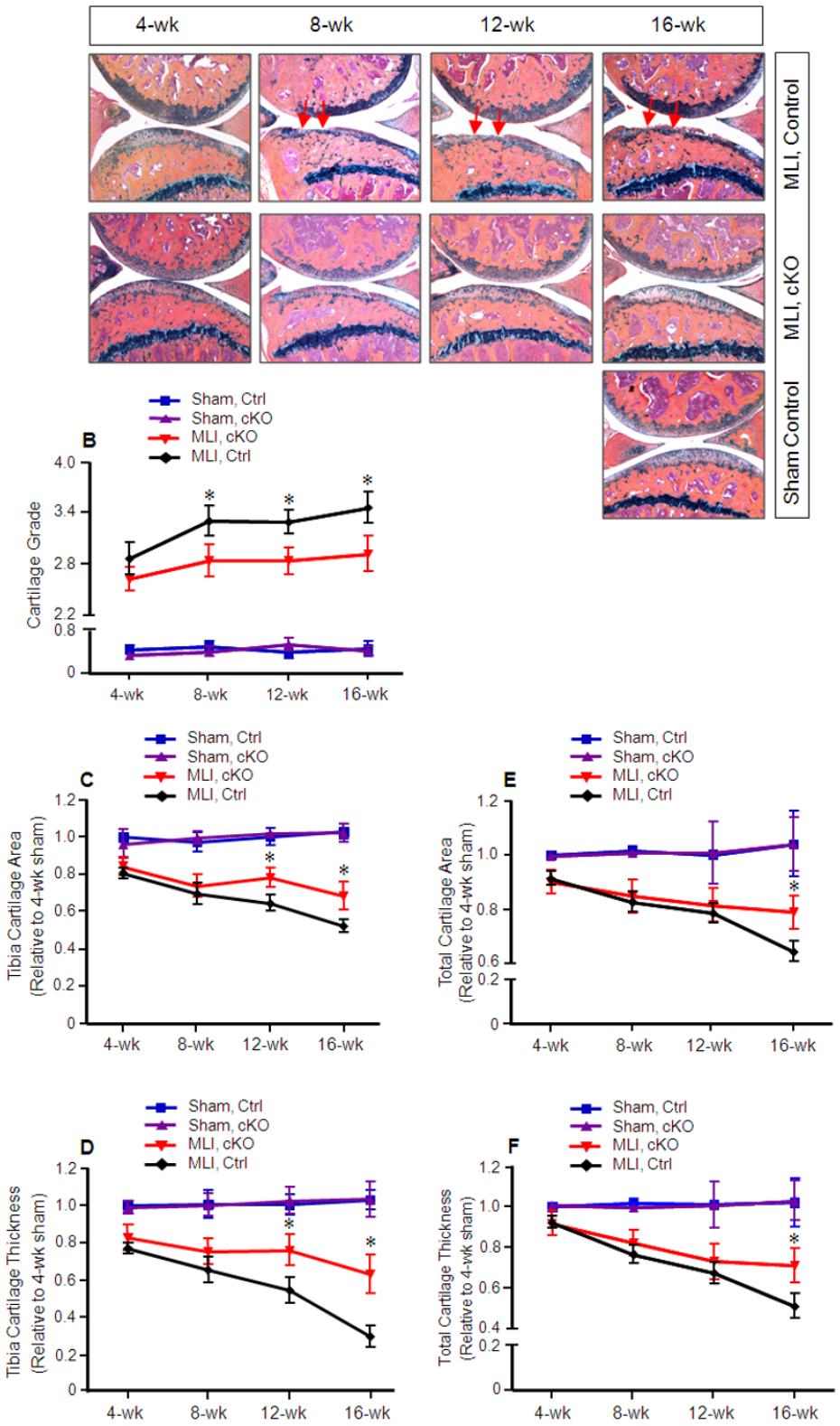

Figure 1 Decelerated osteoarthritis progression in Mmp13 ${ }^{\text {Col2ER }}$ mice. Tamoxifen was administered when matrix metalloproteinase (MMP13) conditional knockout (CKO) mice (Mmp $\left.13^{\text {COIZER }}\right)$ and Cre-negative control mice were two weeks old $(1 \mathrm{mg} / 10 \mathrm{~g}$ body weight, i.p., daily for five days). Meniscal-ligamentous injury (MLI)-induced osteoarthritis (OA) surgery was performed on the right hind-limbs when the mice were 10 weeks old. The left hind-limbs were used as sham controls. (A) Knee joint samples were harvested 4, 8, 12, or 16 weeks post-surgery and Alcian blue/ Hematoxylin/Orange G staining was performed. Histological results showed decreased articular cartilage degradation in Mmp13 Col2ER mice 8, 12 and 16 weeks post-surgery. (B) Histological grading by blinded observers confirmed decreased articular cartilage degradation in Mmp13 ${ }^{\text {COIZER }}$ mice at 8 , 12 , and 16 weeks compared to control Cre-negative mice $\left({ }^{*} P<0.05\right)$. (C) Tibia articular cartilage area was quantified by tracing the Alcian bluepositive area in the proximal tibia. There was no significant difference 4 and 8 weeks post-surgery in Mmp1 ${ }^{\text {COIIER }}$ MLI mice versus Control MLI mice. The tibia cartilage area was increased $21 \%$ in Mmp13 $3^{\text {Col2ER }}$ MLI mice compared to control MLI mice 12 weeks post-surgery $\left({ }^{*} P<0.05\right)$ and increased $31 \% 16$ weeks post-surgery $(* P<0.05)$. (D) Tibia thickness was quantified by tracing the Alcian blue-positive thickness in the center of the tibial plateau. There was no significant difference in tibial cartilage thickness at 4 or 8 weeks post-surgery. Tibia cartilage thickness was increased $29 \%$ in Mmp $13^{\text {COIIER }}$ MLI mice compared to control MLI mice 12 weeks post-surgery $\left({ }^{*} P<0.05\right)$ and increased $50 \% 16$ weeks post-surgery $\left({ }^{*} P<0.01\right)$.

(E) Total articular cartilage area was quantified by tracing the Alcian blue-positive area in both the proximal tibia and distal femur. No significant differences were detected 4,8 , and 12 weeks post-surgery. Total cartilage area increased $18 \%$ in Mmp $13^{\text {Col2ER }} \mathrm{MLI}$ mice compared to control MLI mice 16 weeks post-surgery $\left({ }^{*} P<0.01\right)$. (F) Total cartilage thickness was quantified by tracing the Alcian blue-positive thickness in the center of the proximal tibia and distal femur. No significant differences were detected at 4, 8, and 12 weeks post-surgery. Total cartilage thickness increased 39\% in Mmp13 ${ }^{\text {COIZER }} \mathrm{MLI}$ mice compared to control MLI mice 16 weeks post-surgery $\left({ }^{*} P<0.01\right)$. 
Col2 and proteoglycan loss and chondrocyte apoptosis are reduced in Mmp13 ${ }^{\text {Col2ER }}$ mice

Safranin O/Fast green (SO/FG) staining and IHC of Col 2 and ColX were performed on both $M m p 13^{\text {Col2ER }}$ and control mice eight weeks post-surgery. The results showed greater proteoglycan and Col2 content in the $M m p 13^{\text {Col2ER }}$ mice (Figure $2 \mathrm{~A}-\mathrm{B}$ ). The results of IHC revealed increased ColX in both the $M m p 13^{\text {Col2ER }}$ and
Cre-negative control groups following MLI, but the injury-associated induction was reduced in the $M m p 13^{C o l 2 E R}$ mice (Figure 2C). To investigate chondrocytes apoptosis, we performed TUNEL staining on knee joints eight weeks post-surgery in $M m p 13^{\text {Col2ER }}$ and control mice. Results of TUNEL staining revealed that $28 \%$ of chondrocytes in articular cartilage underwent apoptosis in control mice that received MLI while only
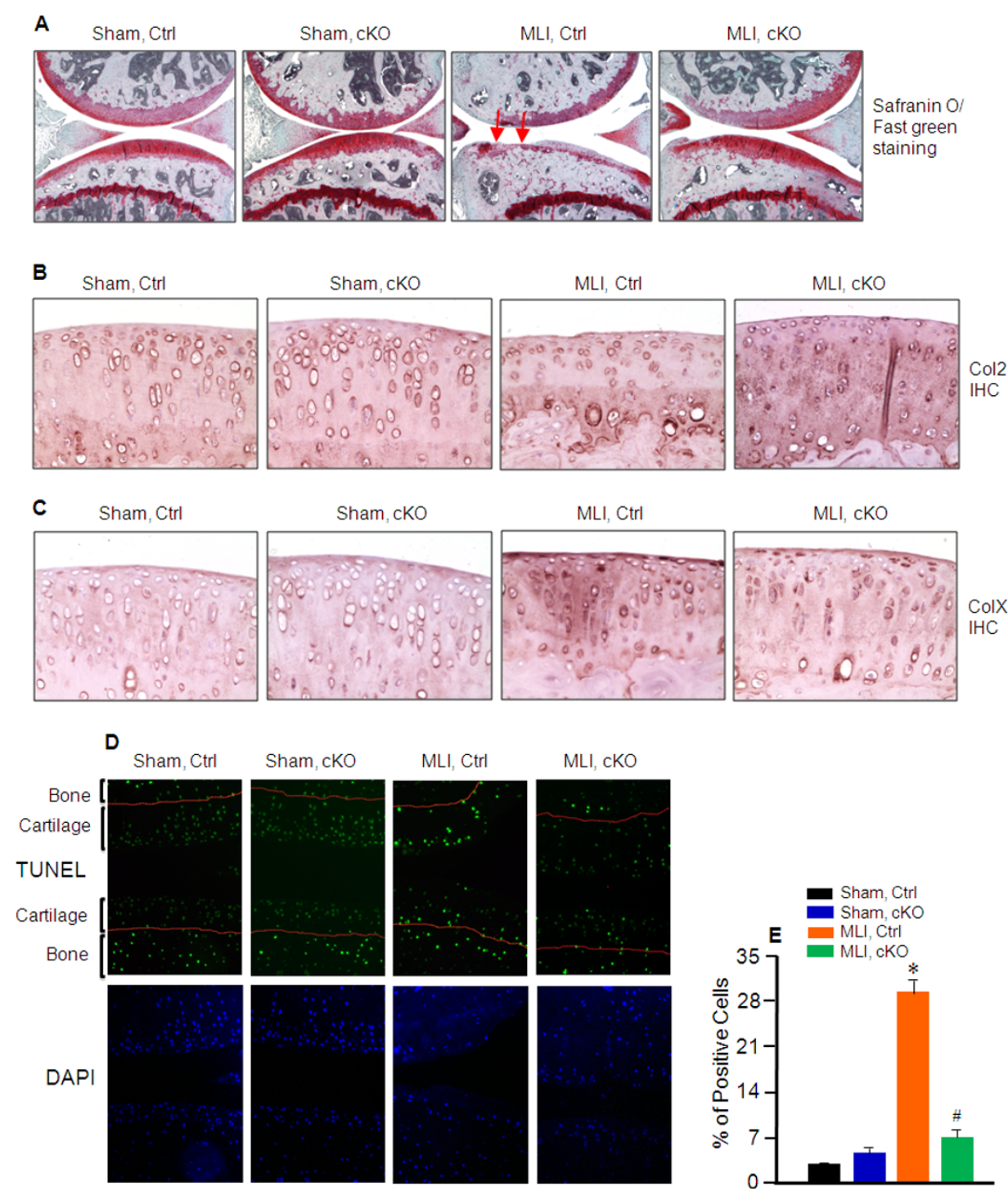

Figure 2 Decelerated osteoarthritis progression in Mmp-13 ${ }^{\text {Col2ER }}$ mice. Tamoxifen was administered when matrix metalloproteinase (MMP13) conditional knockout (CKO) mice $\left(\mathrm{Mmp}^{\mathrm{CO}} \mathrm{COIER}^{2}\right)$ and Cre-negative control mice were two-weeks-old $(1 \mathrm{mg} / 10 \mathrm{~g}$ body weight, intraperitoneal injection, daily for five days). Meniscal-ligamentous injury (MLI) surgery was performed when the mice were 10-weeks-old. Knee joints were harvested eight weeks post-surgery. (A) Safranin O/Fast Green staining was performed to evaluate proteoglycan content. Proteoglycan loss following MLI was reduced in Mmp13 ${ }^{\text {Col2ER }}$ mice. (B) Type II collagen (Col2) loss following MLI was reduced in Mmp13 ${ }^{\text {COIZER }}$ mice.

Immunohistochemistry (IHC) of Col2 was performed to assess its protein level. (C) IHC of ColX was performed to assess its protein level. Type $X$ collagen (CoIX) induction following MLI was reduced in Mmp13 $3^{\text {Col2ER }}$ mice. (D) Terminal deoxynucleotidyl transferase dUTP nick end labeling (TUNEL) staining (in green) and 4',6-diamidino-2-phenylindole (DAPI) nuclear counterstaining (in blue) were performed to assess chondrocyte apoptosis. Chondrocyte apoptosis following MLI was reduced in Mmp 13 ${ }^{\text {Col2ER }}$ mice. (E) Quantification of TUNEL staining positive cells was performed to assess chondrocyte apoptosis. 
$6 \%$ of chondrocytes underwent apoptosis following MLI in Mmp13 ${ }^{\text {Col2ER }}$ mice (Figure 2D and 2E).

\section{CL82198 decelerates MLI-induced OA progression}

We used CL82198, a selective MMP13 inhibitor, to determine if inhibition of MMP13 activity can alter the progression of OA-like degeneration in mice following MLI surgery. Addition of $10 \mu \mathrm{g} / \mathrm{ml}$ of CL82198 together with $5 \mathrm{ng}$ active of MMP13 protein [17] blocked more than $90 \%$ of MMP13 activity in vitro (Figure 3A). In order to mimic the in vivo physiological situation more closely, we isolated primary sternal chondrocytes from three-day-old wild-type pups and treated the cells with bone morphogenetic protein 2 (BMP2) alone, or BMP2 plus 1,5 , or $10 \mu \mathrm{M}$ CL82198 for 60 hours. MMP13 activity in the conditioned media was determined using an MMP13 assay kit. Results showed that after treatment with BMP2, MMP13 activity in conditioned media was significantly increased compared to vehicle control (Figure 3B). In contrast, treatment with $1 \mu \mathrm{M}$ of CL82198 significantly inhibited MMP13 activity (84\% inhibition). More than $90 \%$ of MMP13 activity was inhibited by treatment with 5 or $10 \mu \mathrm{M}$ CL82198 (Figure 3B).

To investigate the efficacy of CL82198 in the inhibition of OA progression, we performed MLI surgery on 10week-old WT mice, followed by i.p.injection of saline, 1, 5, or $10 \mathrm{mg} / \mathrm{kg}$ of CL82198 [18] every other day, beginning one day after surgery, for 12 weeks. Knee joints were harvested 12 weeks post-surgery. Histological results revealed that OA progression, most notably cartilage loss down to and even below the tidemark in saline control mice, was decelerated with 1,5 , or $10 \mathrm{mg} / \mathrm{kg}$ of CL82198 injection, particularly with the $10 \mathrm{mg} / \mathrm{kg}$ dose (Figure 3C). Cartilage grading results were consistent and treatment with 5 and $10 \mathrm{mg} / \mathrm{kg}$ doses of CL82198 had significantly lower scores than the saline control (Figure 3D). Histomorphometric analysis revealed that the articular cartilage area at the proximal tibiae was increased 9\%, 15\% and 43\% (Figure $3 E$ ); total cartilage area was increased $21 \%, 19 \%$ and $38 \%$ (Figure 3F); articular cartilage thickness at the proximal tibiae was increased 11\%, 37\% and 70\% (Figure 3G); and total cartilage thickness was increased $23 \%, 27 \%$ and $50 \%$ (Figure $3 \mathrm{H}$ ) after injection of 1,5 , and $10 \mathrm{mg} / \mathrm{kg}$ of CL82198, respectively.

\section{CL82198 has protective effects on MLI-induced Col2 and proteoglycan loss and chondrocyte apoptosis}

$\mathrm{SO} /$ FG staining was performed to assess proteoglycan content, and IHC was performed to evaluate Col2 and ColX protein levels in the mice with either saline or CL82198 (10 mg/kg) treatment. Results revealed greater proteoglycan and Col2 content in the CL82198 treatment group following MLI surgery compared to the saline treatment group (Figure 4A and 4B). Consistent with these findings, ColX levels were significantly decreased after CL82198 treatment compared to the saline control group (Figure 4C). To assess chondrocyte apoptosis, we performed TUNEL staining on samples from the saline and $10 \mathrm{mg} / \mathrm{kg}$ CL82198 treatment groups. Results of TUNEL staining demonstrated that $34 \%$ of chondrocytes underwent apoptosis in saline control mice following MLI, while only $15 \%$ of chondrocytes underwent apoptosis with CL82198 treatment $(10 \mathrm{mg} / \mathrm{kg}$ ) following MLI (Figure 4D and 4E).

\section{Discussion}

OA is a degenerative joint disease and is the most prevalent form of arthritis. The major symptom of this disease is progressive cartilage break down and eventual complete loss of articular cartilage $[1,19]$. The pathogenesis of OA is not well understood, and there is currently no treatment to alter the progression of OA. In this study, we demonstrated that cartilage-specific deletion of Mmp13 decelerated the progression of $\mathrm{OA}$. We also demonstrated that the MMP13 inhibitor CL82198 prevents OA progression in the MLI-induced murine knee OA model and may, therefore, represent a novel treatment option for OA patients to preserve articular cartilage and joint function instead of simply alleviating OA symptoms as existing treatments do.

Recently, Little et al. showed that global knockout of Mmp13 could prevent articular cartilage erosion [20]. However, there are several limitations related to this study. 1) The Mmp13 global knockout mice have a few abnormalities including focal regions of bony union in growth plates, tendency of metaphyseal flaring and increased trabecular bone mass that may affect OA study [21]. 2) OA is a chronic, progressive disease, and their study only assessed articular cartilage degradation four- and eightweeks post-surgery-induced OA. 3) Their study relied on semi-quantitative histologic methods to characterize OA progression.

To understand better the role of MMP13 in OA development and progression, we generated inducible cartilagespecific Mmp13 ${ }^{\text {Col2ER }}$ mice. Tamoxifen was administered when the mice were two-weeks-old, followed by MLI knee joint surgery to induce OA in Mmp13 ${ }^{\text {Col2ER }}$ and Crenegative control mice $[22,12]$. Our results demonstrate that while articular cartilage is nearly normal four weeks post-surgery in both groups, injured mice progressively develop an OA-like phenotype at the later 8-, 12-, and 16week time points. The histomorphometric data showed that cartilage area and thickness at the proximal tibiae was significantly different at 12 and 16 weeks post-surgery in the $M m p 13^{C o l 2 E R}$ group compared to the control group. However, the total cartilage area and thickness only had a significant difference at 16 weeks post-surgery. This is likely because there was little evidence of cartilage degeneration or loss on the femoral condyle in either 

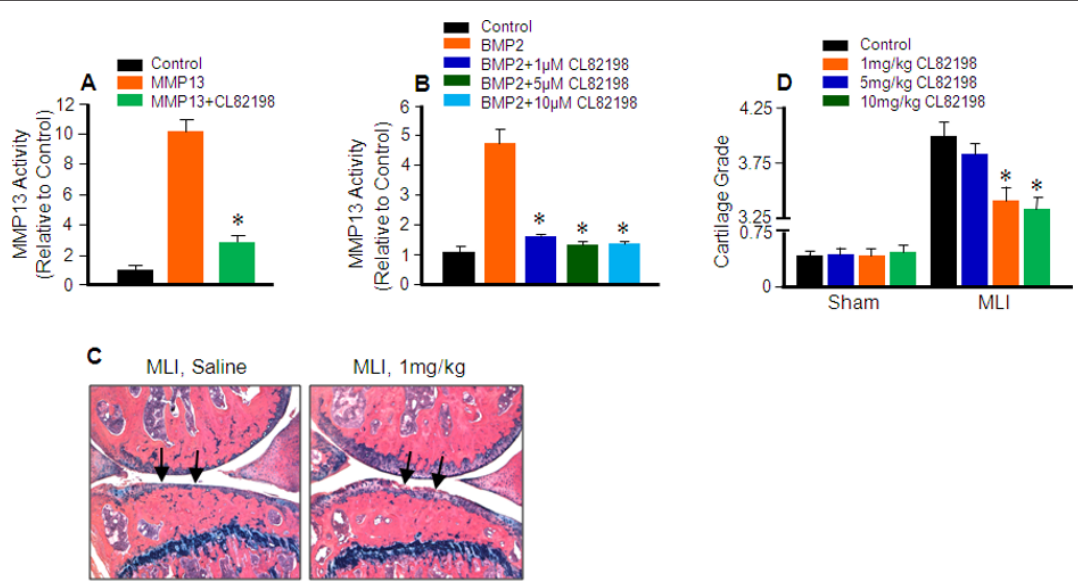

$\mathrm{MLI}, 1 \mathrm{mg} / \mathrm{kg}$
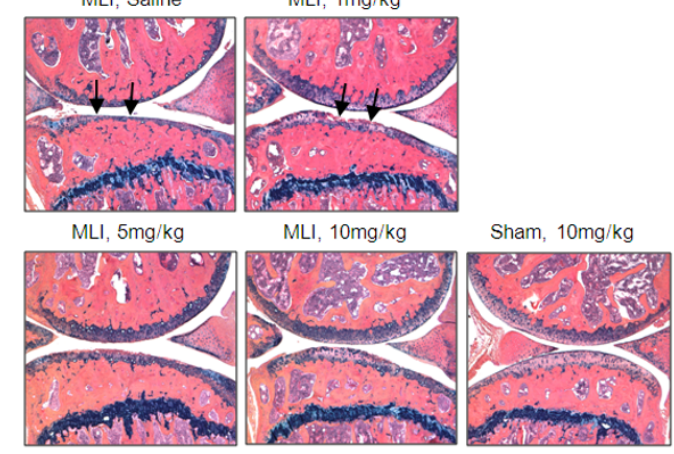

Sham, $10 \mathrm{mg} / \mathrm{kg}$
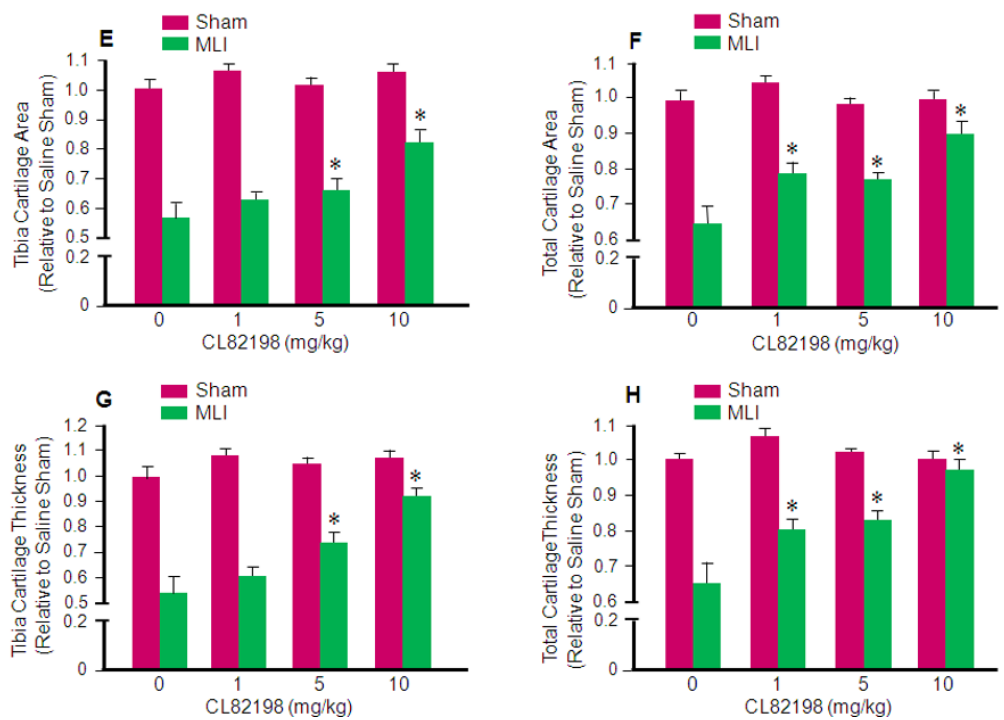

Figure 3 CL82198 prevents and decelerates MLI-induced osteoarthritis progression. (A and B) CL82198 inhibits matrix metalloproteinase (MMP13) activity in vitro. (A) Addition of $5 \mathrm{ng}$ of MMP13, $5 \mathrm{ng}$ of MMP13 and $10 \mu \mathrm{g} / \mathrm{mL}$ of CL82198, or substrate control into a 96-well plate. MMP13 enzyme activity was determined using the SensoLyte 520 MMP13 Assay Kit, and showed that CL82198 could block > 90\% MMP13 activity in vitro. (B) Primary sternal chondrocytes were isolated from three-day-old wild type pups. Cells were plated in 12-well plates and treated with $100 \mathrm{ng}$ bone morphogenetic protein 2 (BMP-2), $100 \mathrm{ng}$ of BMP-2 and $1 \mu \mathrm{M}$ of CL82198, $100 \mathrm{ng}$ of BMP-2 and $5 \mu$ M of CL82198, $100 \mathrm{ng}$ of BMP-2 and $10 \mu \mathrm{M}$ of CL82198, or vehicle control for 60 hours. Cell culture media was collected and MMP13 activity was determined and showed that CL82198 could inhibit > 90\% MMP13 activity produced by BMP-2-treated primary chondrocytes. (C) Inhibition of MMP13 by CL82198 protects against meniscal-ligamentous injury (MLI)-induced osteoarthritis (OA). MLI and sham surgeries were performed on 10-week-old wild type mice. CL82198 was administered to wild type mice one day after surgery by intraperitoneal injection every other day for 12 weeks at doses of 1, 5, or $10 \mathrm{mg} / \mathrm{kg}$ body weight. Normal saline was used as a control. Knee joints were collected, and sectioned 12 weeks post-surgery, and Alcian blue/Hematoxylin/Organge G staining was performed. (D) Histological grading by two blinded observers confirmed decreased articular cartilage degradation in mice treated with 1,5 , and $10 \mathrm{mg} / \mathrm{kg}$ of CL82198 compared to saline control mice $\left.*^{*} P<0.001\right)$. (E) The articular cartilage area at proximal tibiae was quantified by tracing the Alcian blue-positive area in the proximal tibia. Compared to saline MLI, tibial cartilage area was increased 9, 15, and 43\% after treatment with 1, 5, and $10 \mathrm{mg} / \mathrm{kg}$ of CL82198, respectively (*P < 0.05). (F) Total articular cartilage area was quantified by tracing the Alcian blue-positive area in both the proximal tibia and the distal femur. Total cartilage area was increased $21 \%, 19 \%$, and $38 \%$ with treatment of 1,5 , and $10 \mathrm{mg} / \mathrm{kg}$ of CL82198, respectively $\left({ }^{*} P<0.05\right)$. (G) Tibia cartilage thickness was quantified by tracing the Alcian blue-positive thickness in the center of the tibial plateau. Compared to saline treatment, tibia cartilage thickness was increased $11 \%, 37 \%$, and $70 \%$ with treatment of 1,5 , and $10 \mathrm{mg} / \mathrm{kg}$ of CL82198, respectively $\left.{ }^{*} P<0.05\right)$. (H) Total thickness was quantified by tracing the Alcian blue-positive thickness in both the proximal tibia and the distal femur. Total cartilage thickness was increased $23 \%$, $27 \%$, and $50 \%$ after injection of 1,5 , or $10 \mathrm{mg} / \mathrm{kg}$ CL82198, respectively $\left({ }^{*} P<0.05\right)$. 

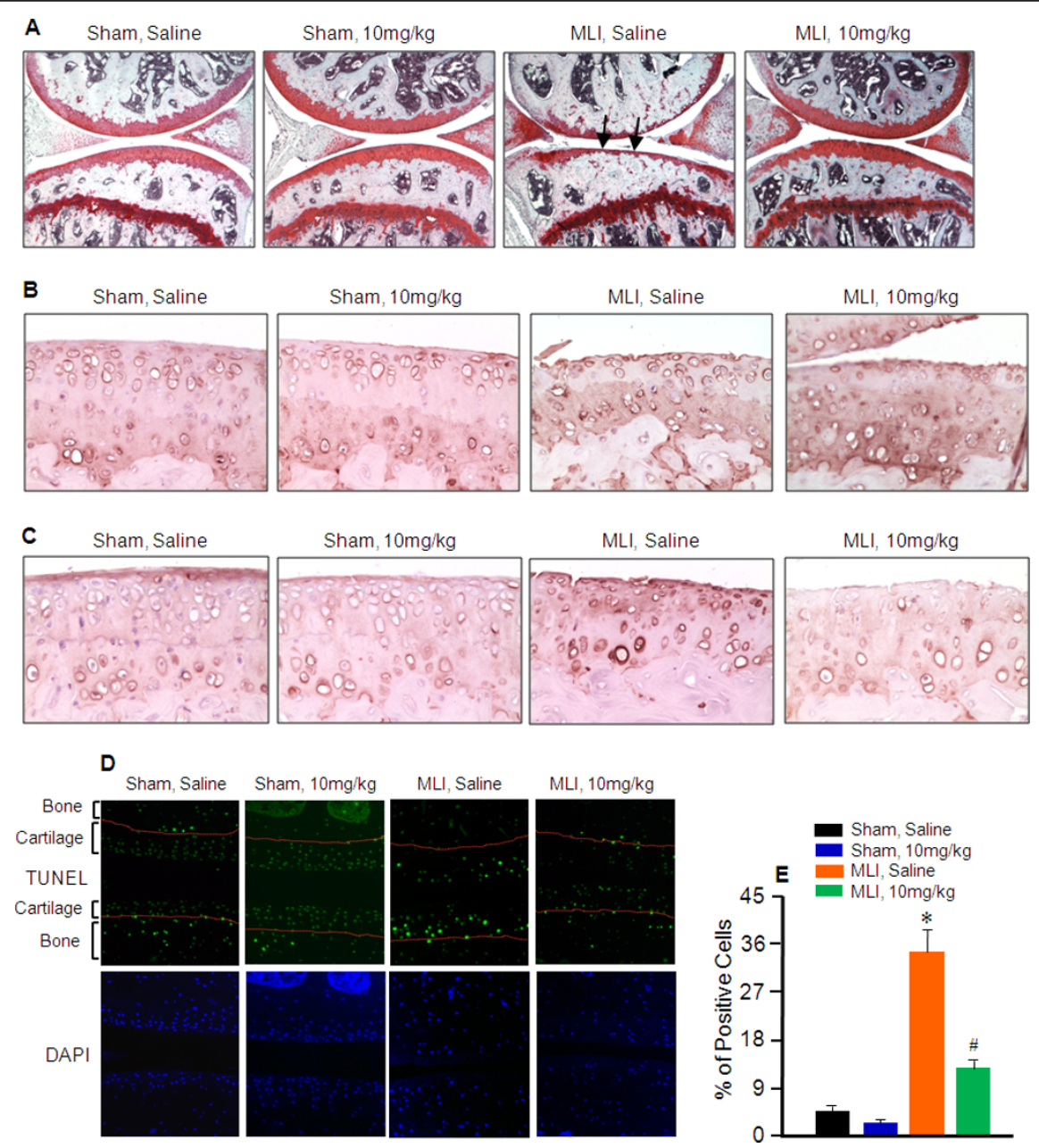

Figure 4 CL82198 prevents and decelerates MLI-induced osteoarthritis progression. Meniscal-ligamentous injury (MLI) and sham surgeries were performed on 10-week-old wild type mice. CL82198 was administered to wild type mice beginning one day after surgery by intraperitoneal injection every other day for 12 weeks at doses of $10 \mathrm{mg} / \mathrm{kg}$ body weight. Normal saline was used as a vehicle control.

(A) Safranin O/Fast green staining was performed to assess proteoglycan content. Proteoglycan loss was reduced following CL82198 treatment. (B) Immunohistochemistry (IHC) of type II collagen (Col2) was performed to assess its protein level. Col2 loss was reduced following CL82198 treatment. (C) IHC of ColX was performed to assess its protein level. Type X collagen (ColX) induction was reduced following CL82198 treatment. (D) Terminal deoxynucleotidyl transferase dUTP nick end labeling (TUNEL) staining (in green) and 4',6-diamidino-2-phenylindole (DAPI) nuclear counterstaining (in blue) were performed to assess chondrocyte apoptosis. Chondrocyte apoptosis was reduced following CL82198 treatment. (E) Quantification of TUNEL staining positive cells was performed to assess chondrocyte apoptosis.

experimental group at 4, 8, 12, or 16 weeks post-surgery, which is consistent with previous studies using this model $[20,12]$.

In addition to morphologic changes in articular cartilage structure, $\mathrm{OA}$ is also characterized by changes in matrix composition as well as changes in articular chondrocyte activity, including inappropriate levels of apoptosis [23-25]. Col2 and proteoglycan content were decreased in both Mmp13 ${ }^{\text {Col2ER }}$ and control groups following MLI surgery. However, cartilage specific deletion of the Mmp13 gene partially prevented Col2 and proteoglycan loss. Deletion of the Mmp13 gene inhibited MLI-induced ColX expression as well. This finding is not unexpected as the cartilage is more intact in $M m p 13^{\text {Col2ER }}$ mice compared to control mice at each time point. TUNEL staining revealed that articular chondrocytes underwent apoptosis in both the control and Mmp $13^{\text {Col2ER }}$ group. However, deletion of the Mmp13 gene dramatically reduced articular chondrocyte apoptosis following MLI, potentially because the more intact cartilage in $M m p 13^{\text {Col2ER }}$ mice may provide chondrocytes with environmental survival cues. Little et $a l$. also showed increased articular chondrocyte apoptosis after surgery, but they did not identify a difference between the control and the Mmp13 KO groups. In their surgery model, they transected the medial meniscotibial ligament to destabilize the medial meniscus. In our 
surgery model, we transected the medial collateral ligament, detached the anterior horn of the medial meniscus from the tibial plateau, and created a tear. Thus, their surgical procedure may elicit a milder OA phenotype than our procedure, and this milder OA phenotype may be the reason for the apparent disparity in the apoptosis results in our and their studies.

Over the past 30 years, several MMP inhibitors have been developed as candidates for the treatment of arthritis, cancer and cardiovascular diseases [26]. However, most of these compounds have failed for a variety of reasons, including non-specificity and toxicity. Currently, no MMP inhibitor has been used in clinics [27]. Recently, Baragi et al. developed an MMP13 inhibitor, ALS 1-0635, and evaluated the efficacy of this compound in a rat OA model [28]. They gave ALS 1-0635 to rats twice-daily beginning one day before surgically-induced OA for three weeks, and found that ALS 1-0635 has chondro-protective effects. However, ALS 1-0635 only had an effect at a dose of 60 $\mathrm{mg} / \mathrm{kg}$. The large dose and frequent administrations of this compound suggest a relatively low specificity for ALS 1-0635 compound.

To explore the therapeutic potential of MMP13 inhibition for OA treatment, we investigated the ability of CL82198, a specific MMP13 inhibitor, to inhibit MMP13 activity in vitro. CL82198 is a chemical compound. Unlike ALS 1-0635 and other MMP13 inhibitors which exert their effects via metal chelation, CL82198 binds to the S1' pocket of MMP13 and showed no effects on MMP-1, -9, or TACE [17]. We found that CL82198 can block more than $90 \%$ of MMP13 activity when it reacts with active MMP13 directly. Since cartilage degeneration and articular chondrocyte dysfunction are hallmarks of $\mathrm{OA}$, we isolated primary sternal chondrocytes from three-day-old WT pups to determine the effect of CL82198 on the activity of MMP13 secreted by chondrocytes undergoing hypertrophy. We treated sternal chondrocytes with BMP2 $(100 \mathrm{ng} / \mathrm{ml})$ for 60 hours to induce hypertrophy and MMP13 production/secretion. Meanwhile, the cells were also treated with CL82198 with BMP2 to inhibit BMP2-induced MMP13 activity. We found that CL82198 inhibited > 90\% of MMP13 activity produced by BMP2-treated primary chondrocytes.

Next, we determined the efficacy of CL82198 in vivo. We performed MLI surgery on 10-week-old WT mice, followed by i.p. injection of saline or 1,5 , or $10 \mathrm{mg} / \mathrm{kg}$ of CL82198 [18] every other day, beginning one day after surgery. Histological data revealed that OA progression was decelerated following CL82198 administration, with the most pronounced effect at $10 \mathrm{mg} / \mathrm{kg}$. Results of cartilage grading and histomorphometric analysis were consistent with histological data and treatment with CL82198 at 5 and $10 \mathrm{mg} / \mathrm{kg}$ doses resulted in significantly lower scores than the saline control.
Consistent with histological and histomorphometric findings, results from SO/FG staining and Col2 and ColX IHC revealed less proteoglycan and Col 2 loss and a decreased ColX level in the CL82198 treatment group. Results of TUNEL staining indicate that about 20\% fewer chondrocytes underwent apoptosis with treatment of CL82198 (10 mg/kg) following MLI compared to saline control mice. Taken together, these results suggest that OA progression is decelerated by the application of a MMP13 inhibitor.

Genetic manipulation is a powerful method to study the genetic causes of diseases and chemical compound delivery in vivo is a practical approach to test the efficacy of drug treatment. Here, we tested the role of MMP13 using Mmp13 cKO mice and using a MMP13 inhibitor in a surgically induced OA mouse model. Our studies suggest that inhibition of MMP13 expression or activity could efficiently prevent and decelerate OA progression. Our study provides initial evidence showing that a MMP13 inhibitor may have potential for the treatment of OA progression although future investigation is still required.

\section{Conclusions}

Our study reveals that cartilage-specific deletion of the Mmp13 gene or inhibition of MMP13 activity decelerates the OA-like phenotype in a surgically induced OA model. Our studies also indicate that MMP13 inhibition could be used as a potential therapeutic strategy for prevention and treatment of $\mathrm{OA}$.

\section{Additional material}

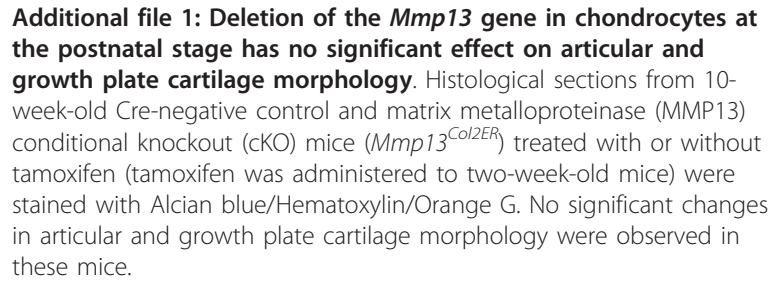

\section{Abbreviations}

AB/HO: Alcian Blue/Hematoxylin/Orange G; ADAMTS: a disintegrin and metalloproteinase with thrombospondin motifs; ANOVA: analysis of variance; BMP-2: bone morphogenetic protein 2; Col2: type II collagen; Col10: type X collagen; DAPI: 4',6-diamidino-2-phenylindole; ELISA: enzyme-linked immunosorbent assay; $\mathrm{H} \& \mathrm{E}$ : hematoxylin and eosin; $\mathrm{HC}$ :

immunohistochemistry; i.p.: intraperitoneal; MLI: meniscal-ligamentous injury; MMP: matrix metalloproteinase; Mmp13 ${ }^{\text {Col2ER }}$ : Col2CreER;Mmp13 fx/fx; OA: osteoarthritis; SO/FG: Safranin O/Fast Green; TUNEL: terminal deoxynucleotidyl transferase dUTP nick end labelling; WT: wild type.

\section{Authors' contributions}

MW and ERS are responsible for study design, data acquisition and analysis and drafting and revising the manuscript. $J$, HJ, and QHK are responsible for data acquisition and analysis. $\mathrm{H}-\mathrm{Jl}$ is responsible for data analysis and interpretation and revision of the manuscript. DC is responsible for study 
design, data analysis and interpretation and revision and final approval of the manuscript. All authors have read and approved the manuscript for publication.

\section{Competing interests}

The authors declare they have no competing interests.

\section{Acknowledgements}

This work was supported by grants R01 AR051189, R01 AR054465 and R01 AR055915 to D.C. from the National Institutes of Health (NIH). H.J. was partially supported by grant No. 81202710 from the National Natural Science Foundation of China and D.C. was partially supported by grant No. Z2111261 from Zhejiang Provincial Natural Science Foundation of China. We gratefully acknowledge the technical expertise of Ryan Tierney and Sarah Mack within the Center for Musculoskeletal Research Histology, IHC, and ISH Core for the processing of all tissue samples.

\section{Author details}

'Department of Orthopedics and Rehabilitation, Center for Musculoskeletal Research, University of Rochester Medical Center, Rochester, NY 14642, USA ${ }^{2}$ Current address: Section of Endocrinology, Department of Internal Medicine, Yale University School of Medicine, 333 Cedar St., PO Box 208020, New Haven, CT 06520, USA. ${ }^{3}$ Current address: Molecular and Cellular Pharmacology, Abbott, 100 Research Drive, Worcester, MA 01605, USA. ${ }^{4}$ Institute of Orthopedics and Traumatology, Zhejiang Chinese Medical University, 548 Binwen Road, Binjiang District, Hangzhou 310053, Zhejiang Province, China. ${ }^{5}$ Department of Biochemistry, Rush University Medical Center, Chicago, 1735 West Harrison Street, IL 60612, USA. 'Liaoning University of Traditional Chinese Medicine, 79 East Chongshan Road, Huanggu District, Shenyang 110847, Liaoning Province, China.

Received: 5 September 2012 Revised: 9 November 2012

Accepted: 3 January 2013 Published: 8 January 2013

\section{References}

1. Goldring MB, Goldring SR: Osteoarthritis. J Cell Physiol 2007, 213:626-634.

2. Wang M, Shen J, Jin H, Im HJ, Sandy J, Chen D: Recent progress in understanding molecular mechanisms of cartilage degeneration during osteoarthritis. Ann N Y Acad Sci 2011, 1240:61-69.

3. Vincenti MP, Brinckerhoff CE: Transcriptional regulation of collagenase (MMP-1, MMP-13) genes in arthritis: integration of complex signaling pathways for the recruitment of gene-specific transcription factors. Arthritis Res 2002, 4:157-164.

4. Shiomi T, Lemaitre V, D'Armiento J, Okada Y: Matrix metalloproteinases, a disintegrin and metalloproteinases, and a disintegrin and metalloproteinases with thrombospondin motifs in non-neoplastic diseases. Pathol Int 2010, 60:477-496.

5. Roach HI, Yamada N, Cheung KS, Tilley S, Clarke NM, Oreffo RO, Kokubun S, Bronner F: Association between the abnormal expression of matrixdegrading enzymes by human osteoarthritic chondrocytes and demethylation of specific $\mathrm{CpG}$ sites in the promoter regions. Arthritis Rheum 2005, 52:3110-3124.

6. Neuhold LA, Killar L, Zhao W, Sung ML, Warner L, Kulik J, Turner J, Wu W, Billinghurst C, Meijers T, Poole AR, Babij P, DeGennaro LJ: Postnatal expression in hyaline cartilage of constitutively active human collagenase-3 (MMP-13) induces osteoarthritis in mice. J Clin Invest 2001, 107:35-44.

7. Glasson SS, Askew R, Sheppard B, Carito B, Blanchet T, Ma HL, Flannery CR, Peluso D, Kanki K, Yang Z, Majumdar MK, Morris EA: Deletion of active ADAMTS5 prevents cartilage degradation in a murine model of osteoarthritis. Nature 2005, 434:644-648.

8. Majumdar MK, Askew R, Schelling S, Stedman N, Blanchet T, Hopkins B, Morris EA, Glasson SS: Double-knockout of ADAMTS-4 and ADAMTS-5 in mice results in physiologically normal animals and prevents the progression of osteoarthritis. Arthritis Rheum 2007, 56:3670-3674.

9. Hunter DJ, Zhang YQ, Niu JB, Tu X, Amin S, Clancy M, Guermazi A, Grigorian M, Gale D, Felson DT: The association of meniscal pathologic changes with cartilage loss in symptomatic knee osteoarthritis. Arthritis Rheum 2006, 54:795-801.

10. Ding C, Martel-Pelletier J, Pelletier JP, Abram F, Raynauld JP, Cicuttini F, Jones G: Meniscal tear as an osteoarthritis risk factor in a largely nonosteoarthritic cohort: a cross-sectional study. J Rheumatol 2007, 34:776-784
11. Clements KM, Price JS, Chambers MG, Visco DM, Poole AR, Mason RM: Gene deletion of either interleukin-1beta, interleukin-1beta-converting enzyme, inducible nitric oxide synthase, or stromelysin 1 accelerates the development of knee osteoarthritis in mice after surgical transection of the medial collateral ligament and partial medial meniscectomy. Arthritis Rheum 2003, 48:3452-3463.

12. Sampson ER, Beck CA, Ketz J, Canary KL, Hilton MJ, Awad H, Schwarz EM, Chen D, O'Keefe RJ, Rosier RN, Zuscik MJ: Establishment of an index with increased sensitivity for assessing murine arthritis. J Orthop Res 2011, 29:1145-1151.

13. Stickens D, Behonick DJ, Ortega N, Heyer B, Hartenstein B, Yu Y, Fosang AJ, Schorpp-Kistner M, Angel P, Werb Z: Altered endochondral bone development in matrix metalloproteinase 13-deficient mice. Development 2004, 131:5883-5895.

14. Chen M, Lichtler AC, Sheu TJ, Xie C, Zhang X, O'Keefe RJ, Chen D: Generation of a transgenic mouse model with chondrocyte-specific and tamoxifen-inducible expression of Cre recombinase. Genesis 2007, 45:44-50.

15. Wang M, Jin H, Tang D, Huang S, Zuscik M, Chen D: Smad1 plays an essential role in bone development and postnatal bone formation. Osteoarthritis Cartilage 2011, 19:751-762.

16. Zhu M, Chen M, Lichtler AC, O'Keefe RJ, Chen D: Tamoxifen-inducible Crerecombination in articular chondrocytes of adult Col2a1CreER ${ }^{T 2}$ transgenic mice. Osteoarthritis Cartilage 2008, 16:129-130.

17. Chen JM, Nelson FC, Levin JI, Mobilio D, Moy FJ, Nilakantan R, Zask A, Powers R: Structure-based design of a novel, potent, and selective inhibitor for MMP-13 utilizing NMR spectroscopy and computer-aided molecular design. J Am Chem Soc 2000, 122:9648-9654.

18. Hernández Ríos M, Sorsa T, Obregón F, Tervahartiala T, Valenzuela MA, Pozo P, Dutzan N, Lesaffre E, Molas M, Gamonal J: Proteolytic roles of matrix metalloproteinase (MMP)-13 during progression of chronic periodontitis: initial evidence for MMP-13/MMP-9 activation cascade. J Clin Periodontol 2009, 36:1011-1017.

19. Alcaraz MJ, Megías J, García-Arnandis I, Clérigues V, Guillén MI: New molecular targets for the treatment of osteoarthritis. Biochem Pharmacol 2010, 80:13-21.

20. Little CB, Barai A, Burkhardt D, Smith SM, Fosang AJ, Werb Z, Shah M, Thompson EW: Matrix metalloproteinase 13-deficient mice are resistant to osteoarthritic cartilage erosion but not chondrocyte hypertrophy or osteophyte development. Arthritis Rheum 2009, 60:3723-3733.

21. Inada M, Wang Y, Byrne MH, Rahman MU, Miyaura C, López-Otín C, Krane SM: Critical roles for collagenase-3 (Mmp13) in development of growth plate cartilage and in endochondral ossification. Proc Natl Acad Sci USA 2004, 101:17192-17197.

22. Glasson SS, Askew R, Sheppard B, Carito BA, Blanchet T, Ma HL, Flannery CR, Kanki K, Wang E, Peluso D, Yang Z, Majumdar MK, Morris EA: Characterization of and osteoarthritis susceptibility in ADAMTS-4knockout mice. Arthritis Rheum 2004, 50:2547-2558.

23. Blanco FJ, Guitian R, Vazquez-Martul E, de Toro FJ, Galdo F: Osteoarthritis chondrocytes die by apoptosis. A possible pathway for osteoarthritis pathology. Arthritis Rheum 1998, 41:284-289.

24. Thomas CM, Fuller CJ, Whittles CE, Sharif M: Chondrocyte death by apoptosis is associated with cartilage matrix degradation. Osteoarthritis Cartilage 2007, 15:27-34.

25. Sharif $M$, Whitehouse A, Sharman P, Perry M, Adams M: Increased apoptosis in human osteoarthritic cartilage corresponds to reduced cell density and expression of caspase-3. Arthritis Rheum 2004, 50:507-515.

26. Peterson JT: The importance of estimating the therapeutic index in the development of matrix metalloproteinase inhibitors. Cardiovasc Res 2006, 69:677-687.

27. Peterson JT: Matrix metalloproteinase inhibitor development and the remodeling of drug discovery. Heart Fail Rev 2004, 9:63-79.

28. Baragi VM, Becher $G$, Bendele AM, Biesinger R, Bluhm H, Boer J, Deng $H$, Dodd R, Essers M, Feuerstein T, Gallagher BM Jr, Gege C, Hochgürtel M, Hofmann M, Jaworski A, Jin L, Kiely A, Korniski B, Kroth H, Nix D, Nolte B, Piecha D, Powers TS, Richter F, Schneider M, Steeneck C, Sucholeiki I, Taveras A, Timmermann A, Van Veldhuizen J, Weik J, Wu X, Xia B: A new class of potent matrix metalloproteinase 13 inhibitors for potential treatment of osteoarthritis: evidence of histologic and clinical efficacy without musculoskeletal toxicity in rat models. Arthritis Rheum 2009, 60:2008-2018. 
doi:10.1186/ar4133

Cite this article as: Wang et al:: MMP13 is a critical target gene during the progression of osteoarthritis. Arthritis Research \& Therapy 2013 15:R5.

Submit your next manuscript to BioMed Central and take full advantage of:

- Convenient online submission

- Thorough peer review

- No space constraints or color figure charges

- Immediate publication on acceptance

- Inclusion in PubMed, CAS, Scopus and Google Scholar

- Research which is freely available for redistribution

Submit your manuscript at 